Original Paper http://ajol.info/index.php/ijbcs http://indexmedicus.afro.who.int

\title{
Diversité biologique et influence des changements climatiques sur la distribution géographique de quelques espèces d'Acanthaceae en Côte d'Ivoire
}

\author{
Ebah Estelle ASSEH ${ }^{1 *}$, Emma AKE-ASSI ${ }^{1,2,3}$ et Kouao Jean $\mathrm{KOFFI}^{4}$ \\ ${ }^{1}$ Laboratoire de Botanique, UFR Biosciences, Université Félix Houphouët-Boigny, Cocody-Abidjan, \\ Côte d'Ivoire. \\ ${ }^{2}$ Centre National de Floristique, 22 BP 582 Abidjan 22, Côte d'Ivoire. \\ ${ }^{3}$ Institut Botanique Aké-Assi d'Andokoi (IBAAN), 08 BP 172 Abidjan 08, Côte d'Ivoire. \\ ${ }^{4}$ Nangui Abrogoua, UFR-SN, 02 BP 801 Abidjan 02, Côte d'Ivoire. \\ *Auteur correspondant; E-mail: assehebah@yahoo.fr ; Tel: (+225) 47784837
}

\section{RESUME}

La présente étude a pour objectif principal d'évaluer la diversité et l'influence des changements climatiques sur la distribution spatiale des espèces de la famille des Acanthaceae en Côte d'Ivoire. Le principe de « Maximum Entropie » a été utilisé pour modéliser les habitats de Dicliptera alternans Lindau, Elytraria ivorensis Dokosi et de Whitfieldia lateritia Hook. sous les conditions climatiques actuelles et futures (horizon 2050/scénario A2). Ces espèces ont été choisies d'une part sur la base du nombre d'occurrences et d'autre part en tenant compte de leur caractère endémique. Les coordonnées de présence des espèces ont été collectées et combinées aux données bioclimatiques extraites de la base de données www.worldbioclim.org. La superficie des zones de distribution potentiellement favorables ou non, a été calculé pour chaque espèce. La recherche documentaire a révélé un total de 106 espèces reparties en 39 genres dont $16 \%$ sont endémiques à la Côte d'Ivoire. 17 espèces à statut particulier dont 9 figurent sur la liste rouge de l'UICN et 8 pour la liste locale des espèces menacées et rares ivoiriennes de Aké-Assi L. Les valeurs de l'AUC (Area Under the Curve) de l'ordre de 0,90 indiquent une excellente qualité des modèles générés pour la distribution des espèces modèles. à l'horizon 2050, les résultats révèlent que la «Saisonnalité de la température (Bio 4) » est la variable environnementale qui a le plus contribué à la prédiction du modèle des espèces Dicliptera alternans Lindau, Elytraria ivorensis Dokosi et Whitfieldia lateritia Hook. Le scénario A2 projette à l'horizon 2050 une réduction de la surface des zones potentiellement favorables des espèces des Dicliptera alternans Lindau, Elytraria ivorensis Dokosi et Whitfieldia lateritia Hook respectivement de 67,66\%, 3,35\% et de 1,17\%. Malgré ces changements observés, les résultats montrent que les aires protégées en Côte d'Ivoire demeurent des refuges sous les conditions environnementales actuelles et futures pour les espèces de la famille des Acanthaceae. Cette étude confirme l'impact avéré des changements climatiques sur la distribution spatiotemporelle des espèces.

(C) 2019 International Formulae Group. All rights reserved

Mots clés: Diversité biologique, changement climatique, Scénario A2, Acanthaceae, Côte d'Ivoire. 


\title{
Biological diversity and influence of the climate changes on the geographical distribution of some species of Acanthaceae in Ivory Coast
}

\begin{abstract}
The object of this study is to estimate the diversity and the influence of the climate changes on spatial of the species of the family of Acanthaceae in Ivory Coast. The principle of «Maximum Entropy» was used to model the habitats of Dicliptera alternans Lindau, Elytraria ivorensis Dokosi and of Whitfieldia lateritia Hook. under the current and future climatic conditions (horizon 2050/scénario A2). These species were selected on the one hand on the basis of number of occurrences and on the other hand by taking account of their endemic nature. The contact of presence of the species was collected and combined with the bioclimatic data extracted the database www.worldbioclim.org. The surface of the potentially favorable zones of distribution or not, was calculated for each species. The document retrieval revealed a total of 106 species left again between 39 kinds and of which $16 \%$ are endemic at the Ivory Coast. 7 species are register on the local list of the endangered species and rare of the Ivory Coast of Aké-Assi L. Les values of the AUC of about 0.90 indicate excellent quality of the models generated for the distribution of the model species. by 2050, the results reveal that the «Seasonal variation of the temperature (Bio 4)» is the environmental variable which contributed the most to the prediction of the model of the species Dicliptera alternans Lindau, Elytraria ivorensis Dokosi and Whitfieldia lateritia Hook. The A2 scenario shows by a 2050 reduction of the surface of the potentially favorable zones of the species will des Dicliptera alternans Lindau, Elytraria ivorensis Dokosi and Whitfieldia lateritia Hook respectively of $67.66 \%, 3.35 \%$ and of $1.17 \%$. In spite of these changes observed, the results show that the protected areas in Ivory Coast remain refuges under the current and future environmental conditions for the species of the family of Acanthaceae. This study confirms the impact proven of the climate changes on the spatiotemporelle distribution of the species.
\end{abstract}

(C) 2019 International Formulae Group. All rights reserved

Keywords: Biological diversity, climate change, A2 Scenario, Acanthaceae, Ivory Coast.

\section{INTRODUCTION}

Les forêts tropicales se caractérisent par une richesse biologique très importante avec des structures et des compositions floristiques variées. Au niveau africain, cette flore se développe dans divers écosystèmes, ce qui induit une flore et une végétation très diversifiée (Gnagbo, 2015). Avec les menaces qui pèsent sur la biodiversité, certaines de ces espèces deviennent de plus en plus rares au niveau de leurs habitats naturels. En dehors des menaces anthropiques, le changement climatique apparait aujourd'hui comme une menace additionnelle pour les populations végétales et animales (Sala et al., 2001). Il est probable que les fluctuations des variables climatiques telles que les précipitations et la température aient une incidence sur la diversité biologique et sur la répartition géographique des habitats favorables aux espèces (IPCC, 2007). La Côte d'ivoire, à l'instar des pays africains, subit les impacts du changement climatique sur la biodiversité. Plusieurs travaux de recherches ont permis de les attester (Kouakou et al., 2012, Sorokoby et al., 2013, Kouamé et al., 2014, Oga et al., 2016). Ces auteurs confirment que la Côte d'Ivoire a subit des variations importantes au niveau des conditions climatiques. Ils prédisent aussi une augmentation de la température et une baisse des précipitations sur la majeure partie du pays. A ce jour, il existe peu d'études sur l'impact des changements climatiques concernant la distribution potentielle des espèces végétales. Certaines études traitent de l'impact des changements climatiques actuelles sur la distribution des Epiphytes (Gnagbo et al., 2015), des Rubiaceae (Tuo et al., 2017), des espèces endémiques (Vroh et al., 2016) au niveau de la Côte d'Ivoire et des espèces consommées par les chimpanzés dans le Parc 
national de Taï (Condé, 2017). Celles relatives à l'impact des conditions climatiques futures portant notamment sur la distribution d'espèces appartenant à un même taxon ou des taxons d'une famille, restent encore à documenter.

Les Acanthaceae sont l'une des familles les plus riches en espèces dans le monde avec environ 3500 espèces regroupées en 229 genres (APG IV, 2016). Elle est constituée de nombreuses espèces ayant des fleurs très colorées. En Côte d'Ivoire, cette famille est classée parmi les familles les plus riches en plantes ornementales (Aké-Assi, 2015) appartenant à la flore naturelle. Par ailleurs, les espèces de cette famille sont utilisées comme plantes médicinales, ornementales et alimentaires (Grubben, 2004). Présente sur toute l'étendue du territoire ivoirien, les espèces de la famille des Acanthaceae tendent à disparaitre de leur milieu naturel à cause des fortes perturbations d'origines anthropiques du milieu naturel forestier (Aké-Assi et al., 2010). C'est dans ce contexte déjà contraignant que s'ajoutent les changements climatiques qui ont une incidence sur la diversité biologique et sur la répartition géographique des habitats favorables aux espèces locales (GIEC, 2007). Il s'avère donc nécessaire d'évaluer les effets probables des changements climatiques sur la distribution géographique de ces espèces locales. Or des études ayant évaluées l'impact potentiel des changements climatiques sur la distribution géographique des aires favorables des espèces en Côte d'Ivoire reste encore à documenter. Ainsi, l'objectif de cette étude est de contribuer à la connaissance de l'impact des changements climatiques sur la répartition géographique des espèces en Côte d'Ivoire au travers de la famille des Acanthaceae. De façon spécifique, il s'agira de relever la diversité (richesse spécifique et endémisme), de modéliser la distribution géographique des aires favorables de ces espèces et d'évaluer l'effet potentiel des changements climatiques à l'horizon 2050 sur l'étendue de ces aires et leur distribution géographique.

\section{MATERIEL ET METHODES Cadre d'étude}

La présente étude a été conduite en Côte d'Ivoire. Localisée en Afrique de l'ouest, la Côte d'Ivoire s'inscrit dans un carré dont les côtes sont situées, d'une part, entre $4^{\circ} 30$ et $10^{\circ} 30$ de latitude Nord et, d'autre part, entre $2^{\circ} 30$ et $8^{\circ} 30$ de longitude Ouest. Le territoire est limité au Sud par l'Océan Atlantique, au Nord par le Mali et le Burkina Faso, à l'Est par le Ghana et, à l'Ouest, par le Libéria et la Guinée. Sa superficie est de l'ordre de 322,500 $\mathrm{Km}^{2}$. L'étude couvre quatre zones climatiques : le climat subéquatorial ; le climat tropical humide ; le climat tropical et le climat de montagne. La végétation de la Côte d'Ivoire est subdivisée en Domaines et Secteurs basés surtout sur les formations végétales dominantes dans le paysage. On distingue ainsi : le domaine guinéen, comprenant, du Sud vers le Nord : le secteur littoral ; le secteur ombrophile ; le secteur mésophile ; le secteur montagnard; et le domaine soudanais, comprenant du Sud vers le Nord : le secteur Sub-soudanais ; le secteur soudanais. Le domaine guinéen est dominé par la forêt dense humide constituée de 2 types principaux de forêt dense humide : la forêt dense humide sempervirente ou ombrophile et la forêt dense humide semi-décidue ou mésophile. Quant au domaine soudanais il est constitué des forêts claires et de savanes. Cependant face à la dégradation galopante $\mathrm{du}$ milieu naturel ivoirien, les aires protégées représentées par les cent quatre-vingt-huit forêts classées, les huit parcs nationaux et les six réserves naturelles sont désormais, les derniers vestiges de la forêt dense ivoirienne (Sangne et al., 2015).

\section{Collecte des données}

Les coordonnées de présence de chaque espèce ont été collectées dans l'Herbier du Centre National de Floristique (C.N.F) de l'Université Félix HouphouëtBoigny, l'Herbier de l'Institut Botanique AkéAssi d'Andokoi (IBAAN), les livres, les données du Global Biodiversity Information Facility (GBIF) sur le site www.gbif.org. et du 
SIG-IVOIRE ont été consultés. Pour certaines récoltes ne disposant pas de coordonnées géographiques, le « Gazetiers», a été utilisé pour identifier leurs coordonnées géographiques. Toutes ces données ont été compilées et ont permis d'avoir une liste de noms d'espèces corrigés avec les occurrences.

Pour établir les modèles de distribution potentielle, nous avons utilisé dix-neuf (19) variables bioclimatiques (Tableau1) téléchargées à partir de la base de données Worldclim version 1.4 via le site www.worldbioclim.org avec une résolution de 2,5 arc-minute au sol. Les conditions climatiques couvrent la période actuelle (1950-2000) et futur (2000-2050). Pour les projections climatiques futures, il existe plusieurs scénarii issus du Rapport spécial du Groupe d'Experts Intergouvernemental sur l'évolution du Climat (GIEC, 2001) sur les scénarios d'émission (SRES). Dans cette étude, Pour tous les modèles, les projections faites pour 2050 ont été utilisées sous le scénario d'émission A2. Ce scénario a été utilisé de préférence car il prédit une situation considérée plus probable pour l'Afrique à l'horizon 2050 (Williams et al., 2007). En effet, le scénario d'émission A2 décrit un monde très hétérogène, une population à fort taux de croissance avec un faible niveau technologique et de développement.

\section{Analyse des données \\ Diversité biologique}

Les différents types biologiques et la répartition phytogéographique des Acanthaceae ont été répertoriés en se référant à la flore de Côte d'Ivoire (Aké Assi, 1984, 2001, 2002). Le coefficient G / E (rapport du nombre de genres sur le nombre d'espèces) a été utilisé pour estimer la diversité floristique. Une faible valeur (proche de 1) de ce coefficient témoigne de la forte diversité de la flore (Tuo et al., 2017).

\section{Espèces modèles}

Pour cette étude, trois espèces ont servi de modèles. Elles ont été choisies en tenant compte d'une part qu'elles ont un nombre d'occurrences $\geq 10$ et d'autre part du statut particulier qu'elles présentent. En effet selon
Gnagbo (2015), le nombre d'occurrence supérieure à dix (10) permet une robustesse du modèle. Ainsi Whitfieldia lateritia Hook. (49 occurrences), Dicliptera alternans Lindau (espèce classée rare selon Aké-Assi, 1998) avec 10 points d'occurrences, et Elytraria ivorensis Dokosi (la seule Acanthaceae endemique à la Côte d'Ivoire) avec 14 points d'occurrences, ont été retenues.

\section{Modélisation et validation du modèle}

La modélisation s'est faite à partir du modèle Maximum Entropie (MaxEnt). C'est un programme qui permet d'estimer et de prédire la distribution spatiale d'une espèce à partir de données de présence de l'espèce en relation avec les conditions environnementales dans la zone d'intérêt (Phillips et al., 2006).

Les dix-neuf variables bioclimatiques actuelles et futures ont été soumises à un test de corrélation. Ce test qui met en évidence les liens existants entre les variables, nous a permis de sélectionner les variables les moins corrélées (coefficient de corrélation inférieur en valeur absolue, à 0,8). Pour Elith et al. (2011), une forte corrélation entre les variables entraine un biais sur les prédictions.

La modélisation s'est faite en introduisant dans l'algorithme de traitement de MaxEnt les coordonnées géographiques de présence de chaque espèce enregistrée dans un fichier au format CSV (Gbesso et al., 2013). Par la suite, les variables bioclimatiques actuelles (1950-2000) et futures (horizon 2050) ont été intégrer dans le même algorithme. En outre, un test de Jackknife a été effectué sur les variables bioclimatiques considérées pour déterminer celles qui contribuent le plus à la modélisation (Fandohan et al., 2013). La performance du modèle a également été évaluée en utilisant la statistique AUC (Area Under the Curve ; Phillips et al., 2006). Pour un modèle généré par MaxEnt, Araújo et al. (2005) recommandent une interprétation de l'AUC selon le Tableau 2.

Le logiciel Arcgis10.1. pour cartographier la distribution géographique actuelle des habitats favorables de l'espèce et celles du futur a été utilisé. La carte du réseau 
national des aires protégées (parcs et réserves nationaux) de la Côte d'Ivoire a été superposée aux cartes de distribution potentielle de chaque espèce modèle issues de MaxEnt.

Les différents niveaux d'habitats de nos espèces modèles ont été classifiés à partir des seuils de probabilités logistiques de présence générée par les modèles. La probabilité logistique d'occurrences d'une espèce est considérée comme indicatrice de la qualité des habitats pour l'espèce. Elle varie entre 0 et 1 (Liu et al., 2013). Pour une valeur de probabilité inférieure à 0,229 , l'habitat est dit "potentiellement non favorable". L'habitat est dit "potentiellement favorable" pour les valeurs supérieure à 0,229 . L'étendue de chaque niveau d'habitat (aire et pourcentage), aussi bien sous les conditions actuelles que futures a été estimée en vue d'évaluer le gain ou la perte dans l'aire potentiellement favorable de l'espèce à l'échelle du pays (Côte d'Ivoire). Cette estimation s'est effectué à partir de l'outil « spatiale analyst » du logiciel ArcGis dans sa version 10.1.

Les taux de changement (Tc) des habitats actuellement favorables en habitats potentiellement non favorables dans le futur et vice versa ont été estimés pour chaque modèle à partir de la formule suivante (Toyi et al., 2013) :

$$
\mathrm{Tc}=((\mathrm{A} 2-\mathrm{A} 1) / \mathrm{A} 1) \times 100
$$

Dans cette étude, A1 et A2 sont respectivement la superficie initiale (condition climatique actuelle) et finale (condition climatique future) de présence de l'espèce Les valeurs positives de Tc indiquent un gain de la surface de l'habitat tandis que les valeurs négatives correspondent à une perte de surface.

Tableau 1: Variables bioclimatiques utilisées pour le test de Maxent (Guarino et al., 2002).

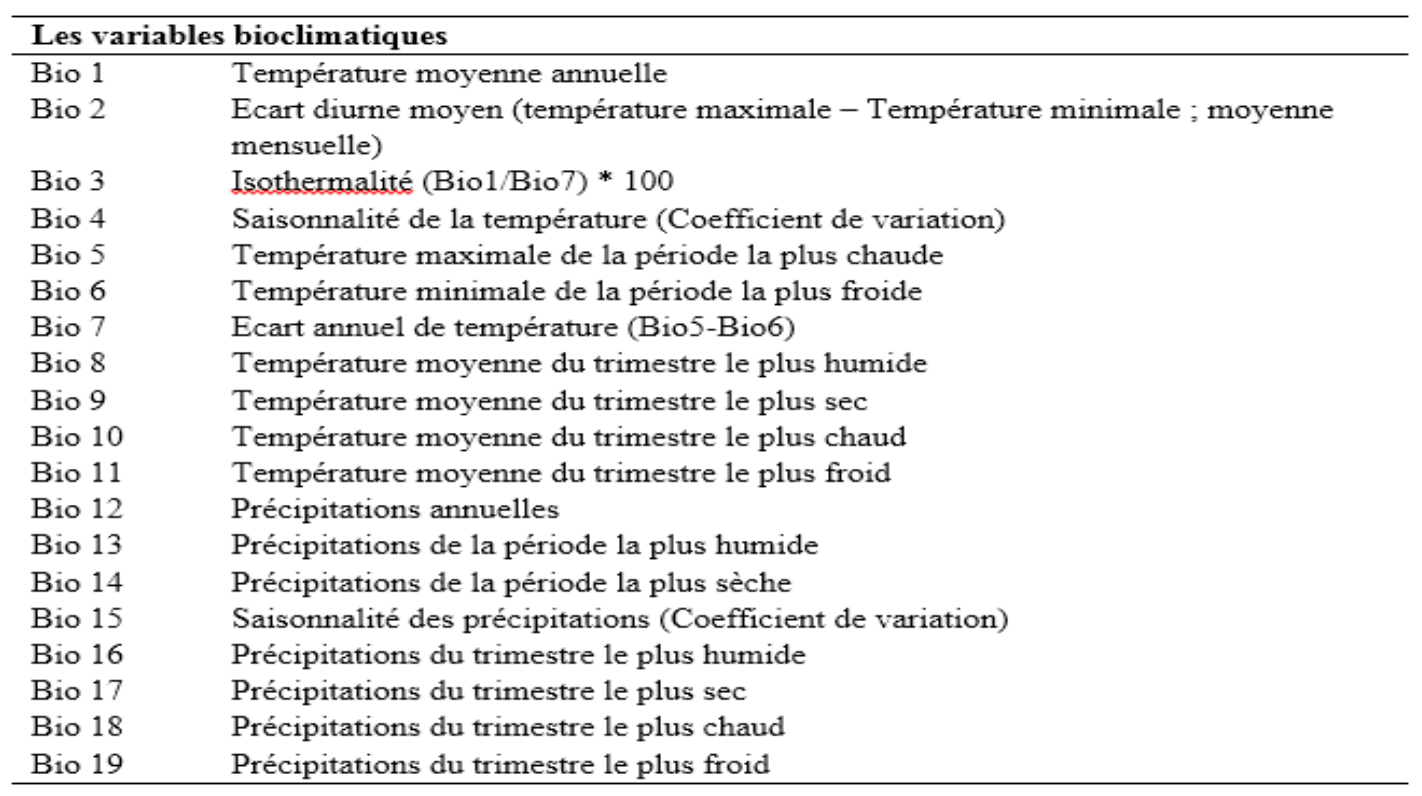

Tableau 2: Validité du test de Maxent suivant les valeurs de l'AUC (Araújo et al., 2005).

\begin{tabular}{ll}
\hline Naleurs & Interprétations \\
\hline $0,90<$ AUC $<1,00$ & Excellente \\
$0,80<\mathrm{AUC}<0,90$ & Bonne \\
$0,70<\mathrm{AUC}<0,80$ & Acceptable \\
$0,60<\mathrm{AUC}<0,70$ & Mauvaise \\
$0,50<\mathrm{AUC}<0,60$ & Invalide \\
\hline
\end{tabular}




\section{RESULTATS \\ Diversité floristique}

Un total de 106 espèces regroupées en 39 genres a été inventorié. Les genres les plus représentés en espèces sont le genre Justicia Retz (12,96\%); Thunbergia Retz. (7,40\%); Barleria (5,55\%); Hygrophila $(5,55 \%)$ et Asystasia (5,55\%), Figure (1). Les nanophanérophytes (np) avec $62 \%$ des espèces sont le type biomorphologique le plus représenté. Les lianes nanophanérophytes (Lnp), les nanophanerophytes hémicryptophytes pyrophytique (npHpy) et les térophytes (Th) sont les moins représentés avec 3\% des espèces chacun (Figure 2). Les données indiquent que $42 \%$ des espèces de la famille des Acanthaceae appartiennent à Guinéo-Congolaise (GC). Les espèces endémiques du bloc forestier à l'Ouest africain et les espèces Guinéo-CongolaisesSoudano-Zambéziennes (GC-SZ) représentent respectivement $13 \%$ et $19 \%$ (Figure 3). Dans cette base de données, 15 espèces (soit $1 \%$ ) sont endémiques de la Côte d'Ivoire parmi lesquelles, Elytraria ivorensis Dokosi est la seule espèce des Acanthaceae endémique à la Côte d'Ivoire (GCi) (Tableau 3). Dans cette famille neuf espèces figurent sur la liste rouge de l'UICN (2016) et sept pour la liste locale des espèces menacées et rares ivoiriennes de Aké-Assi (1998), Tableau 4. Le coefficient générique de la famille des Acanthaceae donné par le nombre de genre sur celui des espèces est de 0,37 soit $37 \%$.

\section{Contribution des variables de la modélisation et validation des modèles}

L'analyse des corrélations a permis de sélectionner les variables environnementales les moins corrélées $(r<0,80)$ pour l'exécution des modèles (Tableau 5). Il s'agit de Bio 1 (Température moyenne annuelle), de Bio 2 (Ecart diurne moyen : température maximale - Température minimale ; moyenne mensuelle), Bio 3 (Isothermalité (Bio1/Bio7) * 100), Bio 13 (Précipitations de la période la plus humide), Bio 15 (Saisonnalité des précipitations :Coefficient de variation) Bio 16 (Précipitations du trimestre le plus humide), Bio 18 (Précipitations du trimestre le plus chaud) et Bio 19 (Précipitations du trimestre le plus froid) sous les conditions climatiques actuelles. Pour ce qui est des conditions climatiques futures ce sont les variables Bio 4 (Saisonnalité de la température (Coefficient de variation)), Bio 6 (Température minimale de la période la plus froide) et Bio 13 (Précipitations de la période la plus humide). Le tableau présente également la contribution des variables à l'édification des différents modèles. Le modèle a généré des valeurs de l'AUC qui varient de 0,918 à 0,988 pour les conditions climatiques actuelles et de 0,923 à 0,985 dans les conditions climatiques futures de 2050, sous le scénario A2 (Tableau 6).

\section{Distribution actuelle et future des habitats favorables aux espèces modèles Distribution de Dicliptera alternans Lindau}

Les résultats des différentes simulations montrent pour la période 19502000 que, la zone potentiellement favorable de l'espèce couvre environ $12,12 \%$ du territoire soit $39105,89 \mathrm{~km}^{2}$. Cette zone potentiellement favorable se localise dans le Sud-ouest principalement dans le Parc National de Taï (PNT). Elle atteint aussi le Centre de la Côte d'Ivoire dans les environs du Parc National de la Marahoué. (Figure 4). La zone potentiellement non favorable quant à elle, occupe $283357,11 \mathrm{~km}^{2}$ soit $87,8 \%$ du territoire national (Tableau 7). Pour l'exécution $\mathrm{du}$ modèle la variable bioclimatique «Isothermalité (Bio1/Bio7) * 100 » est la variable ayant la plus forte contribution $(81,5 \%)$ dans l'édification du modèle de distribution de D. alternans (Figure 5).

Avec les projections bioclimatiques à l'horizon 2050, D. alternans perdra près de $67,66 \%$ (Tableau 7) des zones qui lui sont actuellement favorables. Cette zone s'observera uniquement dans le Parc National de Taï (PNT), Figure 4. «La Saisonnalité de la température, (Bio 4) », est la principale variable qui contribue majoritairement à la 
prédiction de la zone potentiellement favorable de l'espèce (Figure 5).

\section{Distribution de Elytraria ivorensis Dokosi}

L'analyse des habitats donne une superficie de 63309,07 $\mathrm{km}^{2}$ (soit 19,63\%) comme zone de distribution potentiellement favorable à l'espèce pour les conditions actuelles (Tableau 7). Cette zone se situe dans le Sud-ouest au sein du Parc National de Taï et aux environs et dans le Sud-est de la Côte d'Ivoire dans les environs de la Réserve Naturelle des Îles Ehotilés (Figure 6). Quant à la zone potentiellement non favorable à la distribution de l'espèce, elle s'étend sur $259153,93 \mathrm{~km}^{2}$ soit $80,36 \%$ du térritoire ivoirien. «Ecart diurne moyen (Bio 2)», $(72,4 \%)$ et «Isothermalité (Bio1/Bio7) * 100, (Bio 3)», (24,7) sont les variables bioclimatiques ayant le plus contribué à l'édification du modèle de distribution de $E$. ivorensis pour les conditions climatiques actuelles (Figure 7). A l'horizon 2050, le scénario $\mathrm{A} 2$ projette un accroissement de près de $0,82 \%$ des zones potentiellement nonfavorables à l'espèce par perte des zones potentiellement favorables à leur profit (Tableau 7). Elle est passée de $259153,93 \mathrm{~km}^{2}$ (conditions climatiques actuelles) à 261277,18 $\mathrm{km}^{2}$ (conditions climatiques futures). Le scénario prédit également que les zones potentiellement favorables à E. ivorensis au Sud-est ne le seront plus (Figure 6). Les variables environnementales «Saisonnalité de la température (Bio 4) » $(84,4 \%)$ et «
Température minimale de la période la plus froide (Bio 6 », (15,5\%) une meilleure contribution à l'édification du modèle (Figure 7).

\section{Distribution de Whitfieldia lateritia Hook.}

La carte de distribution actuelle (19502000) montre que les zones de distribution potentiellement favorables à $W$. lateritia Hook. sur le territoire national sont le Parc National de Taï (PNT) au Sud-ouest et dans l'ouest au niveau du Mont Nimba (Figure 8). Ces zones représentent $12972,91 \mathrm{~km}^{2}$ soit une couverture de 4\% (Tableau 7). Les «Précipitations du trimestre le plus chaud (Bio 18)», $(32,5)$; «Précipitations du trimestre le plus froid (19), (27,7\%), «Précipitations de la période la plus humide (Bio 13)», $(24,6 \%)$ et «Isothermalité (Bio 3)», (11,6\%) sont les variables bioclimatiques ayant le plus participé à la prédiction des zones d'occupation de l'espèce (Figure 9). Pour ce qui est des projections futures (période 20002050), les zones potentiellement favorables à l'espèce connaitront une légère baisse de l'ordre de $1,17 \%$ de leur couverture (Tableau 7). Cependant la localisation de ces zones favorables demeurent les mêmes. Les variables prédisant au mieux la distribution de W. lateritia sont «Saisonnalité de la température (Bio 4)», (41,9\%) ; «Précipitations de la période la plus humide (Bio 13)», $(28,5 \%)$ et «Précipitations du trimestre le plus froid (Bio 19)», $(25,4 \%)$, (Figure 9).

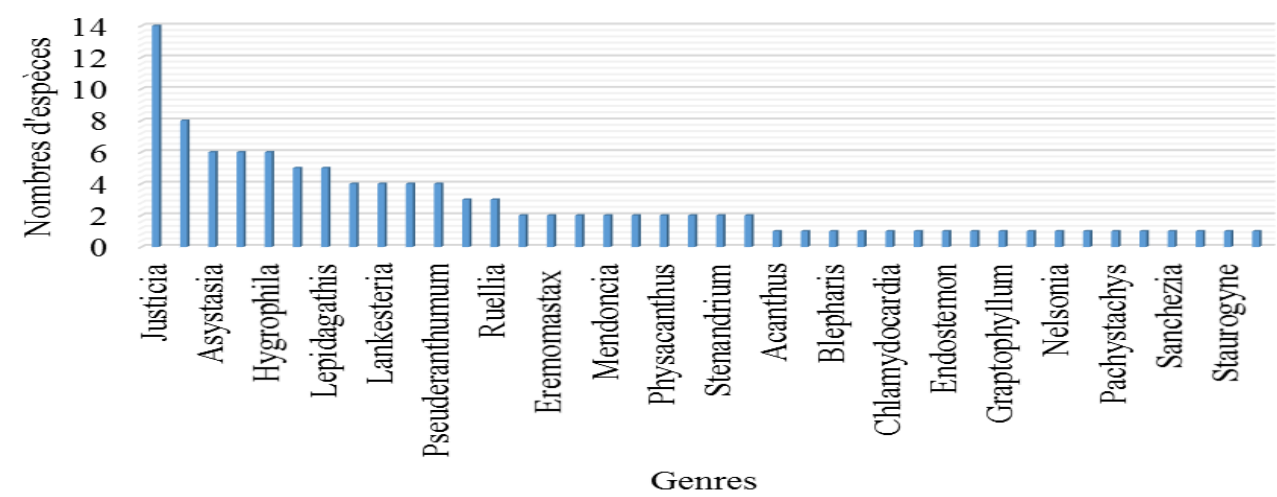

Figure 1: Histogramme de la richesse spécifique par genre de la famille des Acanthaceae recensées en Côte d'Ivoire. 


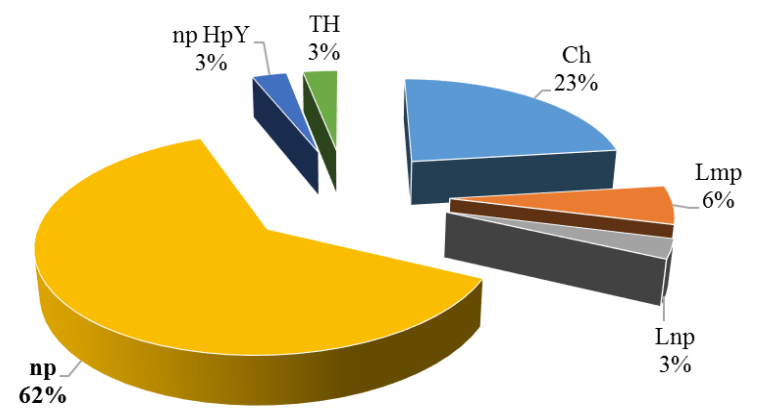

Figure 2: Spectre des types biomorphologiques des espèces recensées.

Légende: $\mathrm{mp}$ : microphanérophytes ; $\mathrm{np}$ : nanophanérophytes ; Lmp: microphanérophytes lianescentes ; Ch : Chaméphytes; Th : thérophytes, Lnp: nanophanérophytes lianescentes ; Lmp : microphanérophytes lianescentes ; npHpy: nanophanérophytes hémicryptophytes. Pyrophytique.

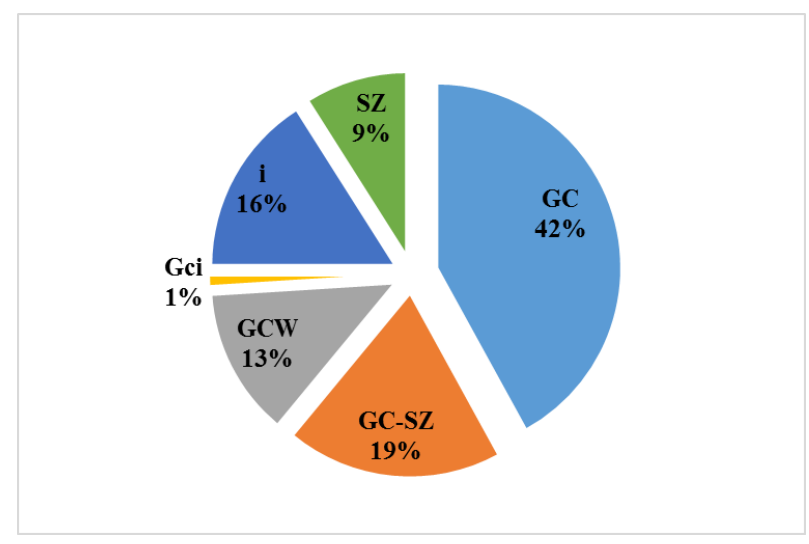

GC: Taxon de la région Guinéo-Congolaise ; GCW : espèces endémiques du bloc forestier à l'Ouest du Togo; GCI : espèces endémiques de la Côte d'Ivoire) ; SZ : Taxon de la région

Soudano-Zambézienne ; GC-SZ:Taxon de la zone de transition entre les régions Guinéo-Congolaise et SoudanoZambézienne; i: Taxon exotique introduit.

Figure 3: Spectres des affinités chorologiques de l'ensemble des espèces inventoriées.

Tableau 3: Effectif des espèces des zones endémiques.

\section{Nombre d'échantillons}

\section{$\mathbf{N}^{\circ} \quad$ Espèces}

1 Brachystephanus jaundensis subsp. nimbae (Heine) I. Darbysh.

2 Elytraria ivorensis Dokosi

3 Heteradelphia paulojaegeria Heine

4 Hygrophila odora (Nees) T. Anderson

5 Justicia grandis (T. Anderson) Vollesen

6 Justicia guineensis (Heine) W.D. Hawth.

7 Lankesteria hispida (Willd.) T. Anderson

8 Mendoncia combretoides (A. Chev.) Benoist

Gci GCW GCW-S

3

14 
9 Rhinacanthus virens var. Obtusifolius (Nees) Milne-Redhead 11

10 Rungia eriostachya Hua

11 Staurogyne capitata E. A. Bruce 23

$\begin{array}{ll}12 \text { Sclerochiton vogelii (Nees) T. Anderson } & 15\end{array}$

13 Stenandrium buntingii (S. Moore) Vollesen $\quad 15$

14 Thomandersia anachoreta Heine $13 \quad 13$

15 Whitfieldia colorata C.B. Clarke ex Stapf $12 \quad 12$

16 Whitfieldia lateritia Hook. $\quad 49 \quad 49$

Légende : GCi : espèce endémique à la Côte d'Ivoire ; GCW : espèces endémiques du bloc forestier Ouest africain ; GCW-S : espèces endémiques du bloc forestier Ouest africain, principalement dans la limite est le fleuve Sassandra situé dans les forêts hygrophiles à l'ouest de la Côte d'Ivoire.

Tableau 4 : liste des espèces à statut particulier.

\begin{tabular}{cll}
\hline \multicolumn{1}{c}{ Listes } & \multicolumn{1}{c}{ Espèces } & Statut \\
\hline UICN (2016) & Stenandrium guineense (Nees) Vollesen & LC \\
\hline & Brillantaisia lamium (Nees) Benth. & LC \\
& Dicliptera eliotti C. B. Cl. & LC \\
& Hygrophila senegalensis (Nees) T. Anders. & LC \\
& Phaulopsis imbricata (Forssk.) Sweet & LC \\
& Physacanthus nematosiphon (Lindau) Rendle \& Britten & DD \\
& Rhinacanthus virens (Nees) MiIne-Redh. var. virens & LC \\
& Ruellia primuloides (T. Anderson ex Benth.) Heine & LC \\
& Sclerochiton vogelii (Nees) T. Anderson & LC \\
Aké-Assi (1998) & Brillantaisia madagascariensis T. Anderson ex Lindau & PRE \\
& Chlamydocardia buettneri Lindau & PDE \\
& Dicliptera alternans Lindau & PRE \\
& Dicliptera laxispica Lindau & PRE \\
& Heteradelphia paulojaegeria Heine & PRE \\
& Sclerochiton vogelii (Nees) T. Anderson & PRE \\
& Thunbergia affinis S.Moore & PRE \\
\hline
\end{tabular}

Légende : LC : préoccupation mineure; DD : données insuffisantes PRE : Plantes rares, devenues rares et en voie d'extinction ; PDE : plantes disparues et en voie d'extinction.

Tableau 5: Variables environnementales sélectionnées et leur contribution au modèle.

\begin{tabular}{|c|c|c|c|c|c|c|c|}
\hline \multirow[b]{2}{*}{ Code } & \multirow[b]{2}{*}{ Dénomination de la variable } & \multicolumn{2}{|c|}{$\begin{array}{l}\text { Dicliptera } \\
\text { alternans Lindau }\end{array}$} & \multicolumn{2}{|c|}{$\begin{array}{l}\text { Elytraria } \\
\text { ivorensis Dokosi }\end{array}$} & \multicolumn{2}{|c|}{$\begin{array}{l}\text { Whitfieldia_lateritia_ } \\
\text { Hook. }\end{array}$} \\
\hline & & Actuel & $\begin{array}{l}\text { horizon } \\
2050\end{array}$ & Actuel & $\begin{array}{l}\text { horizon } \\
2050\end{array}$ & Actuel & $\begin{array}{l}\text { horizon } \\
2050\end{array}$ \\
\hline Bio 1 & Température moyenne annuelle & 0 & 0 & 0 & 0 & 0,4 & 0 \\
\hline Bio 2 & $\begin{array}{l}\text { Ecart diurne moyen } \\
\text { (température maximale - } \\
\text { Température minimale } \\
\text { moyenne mensuelle) }\end{array}$ & 0,4 & & 72,4 & & 0,2 & \\
\hline
\end{tabular}


Bio 3 Isothermalité (Bio1/Bio7) * $100 \quad \mathbf{8 1 , 5}$

24,7

11,6

Bio Saisonnalité de la température

78,8

41,9

4 (Coefficient de variation)

Bio 6 Température minimale de la

10,5

15,5

période la plus froide

Bio Précipitations de la période la

0,2

1,3

0

0

$24,6 \quad 28,5$

13 plus humide

Bio Saisonnalité des précipitations

0

15 (Coefficient de variation)

Bio Précipitations du trimestre le

2,9

1,8

0

16 plus humide

Bio Précipitations du trimestre le

12,2

0

3

18 plus chaud

Bio Précipitations du trimestre le

19 plus froid

2,8

9,3

0

32,5

Tableau 6: Différentes valeurs de l'AUC issues des résultats de la modélisation.

\begin{tabular}{lll}
\hline Taxons & AUC (condition actuelle) & AUC (horizon 2050) \\
\hline Dicliptera alternans Lindau & 0,988 & 0,985 \\
$\begin{array}{l}\text { Elytraria ivorensis Dokosi } \\
\text { Whitfieldia_lateritia_ }\end{array}$ & 0,918 & 0,923 \\
Hook. & 0,981 & 0,959 \\
\hline
\end{tabular}

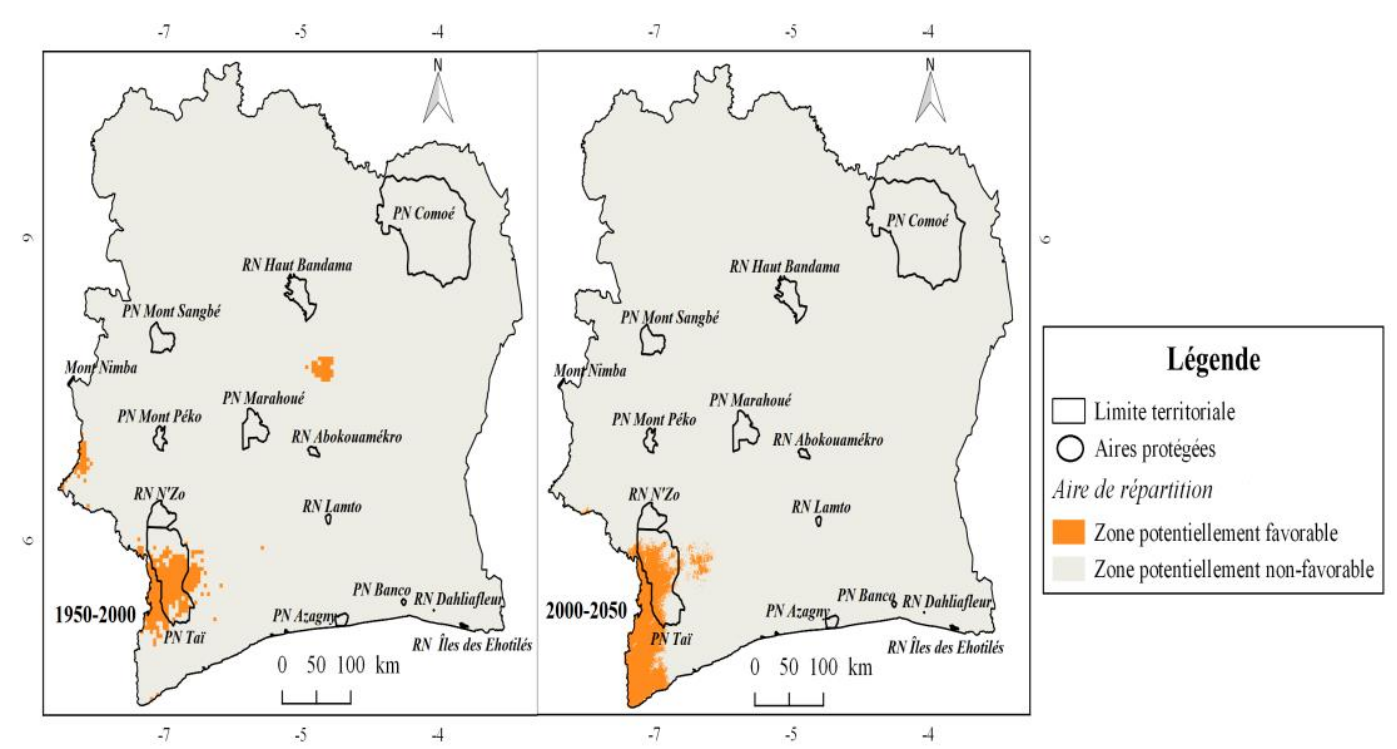

Figure 4: Distribution potentielle actuelle et future de Dicliptera alternans Lindau en Côte d'Ivoire. 
Tableau 7: Dynamique des zones de distribution potentielles des espèces modèles.

\begin{tabular}{|c|c|c|c|c|c|c|c|}
\hline \multirow[t]{2}{*}{ Espèces } & \multirow[t]{2}{*}{ Période } & \multicolumn{2}{|c|}{$\begin{array}{l}\text { Zone potentiellement } \\
\text { favorable }\end{array}$} & \multirow{2}{*}{$\begin{array}{l}\text { Taux de } \\
\text { changement } \\
(\%)\end{array}$} & \multicolumn{2}{|c|}{$\begin{array}{l}\text { Zone potentiellement } \\
\text { non-favorable }\end{array}$} & \multirow{2}{*}{$\begin{array}{l}\text { Taux de } \\
\text { changement } \\
(\%)\end{array}$} \\
\hline & & $\begin{array}{l}\text { Superficie } \\
\left(\mathbf{k m}^{2}\right)\end{array}$ & $\begin{array}{l}\% \text { de } \\
\text { couverture }\end{array}$ & & $\begin{array}{c}\text { uperficie } \\
\left(\mathbf{k m}^{2}\right)\end{array}$ & $\begin{array}{l}\% \text { de } \\
\text { couverture }\end{array}$ & \\
\hline Dicliptera & Actuel & 39105,89 & 12,12 & & 283357,11 & 87,8 & \\
\hline alternans & 2050 & 12646,94 & 3,92 & $-67,66$ & 309816,06 & 96,07 & $+9,34$ \\
\hline Lindau & & & & & & & \\
\hline Elytraria & Actuel & 63309,07 & 19,63 & & 259153,93 & 80,367 & \\
\hline ivorensis & 2050 & 61185,82 & 18,97 & $-3,35$ & 261277,18 & 81,02 & $+0,82$ \\
\hline Dokosi & & & & & & & \\
\hline Whitfieldia & Actuel & 12972,91 & 4,02 & & 309490,09 & 95,97 & \\
\hline lateritia Hook & 2050 & 12820,91 & 3,97 & $-1,17$ & 309642,09 & 96,024 & $+0,04$ \\
\hline
\end{tabular}

Le signe (-) indique une perte d'habitat favorable et le signe (+) indique un gain.
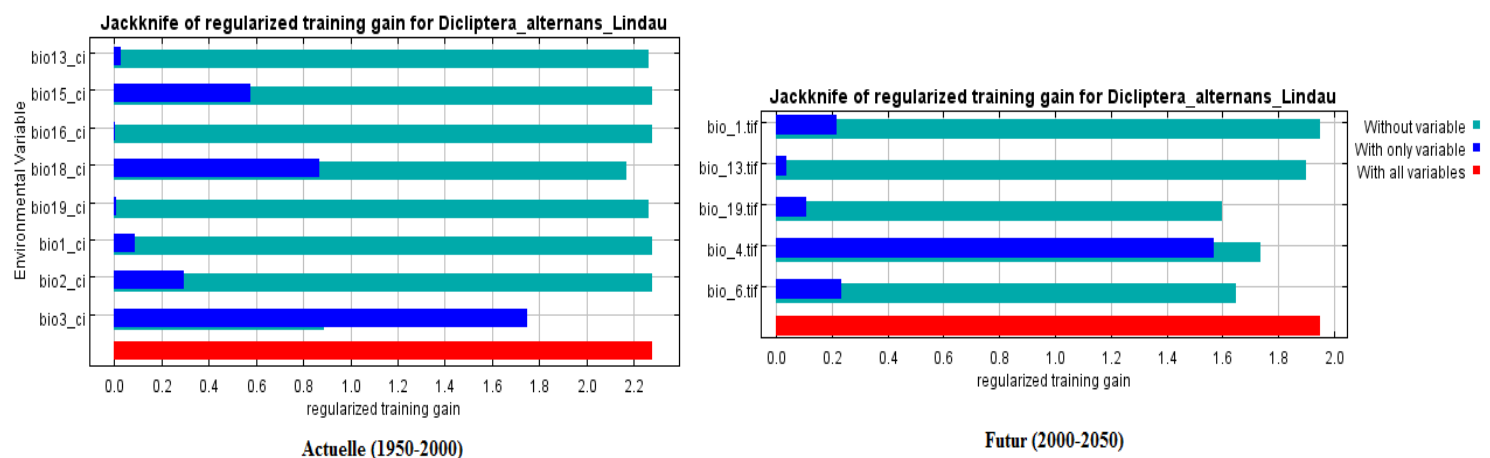

Légende : La figure présente en ordonnées les variables environnementales et en abscisses leurs contributions au calibrage du modèle. La bande devant chaque variable indique les performances du modèle lorsque ce dernier est tourné avec ladite variable seule (bleue) ou sans elle (verte). La bande rouge indique les performances du modele tourné avec toutes les variables.

Figure 5: Résultat du test de Jackniffe sur les variables bioclimatiques du modèle.

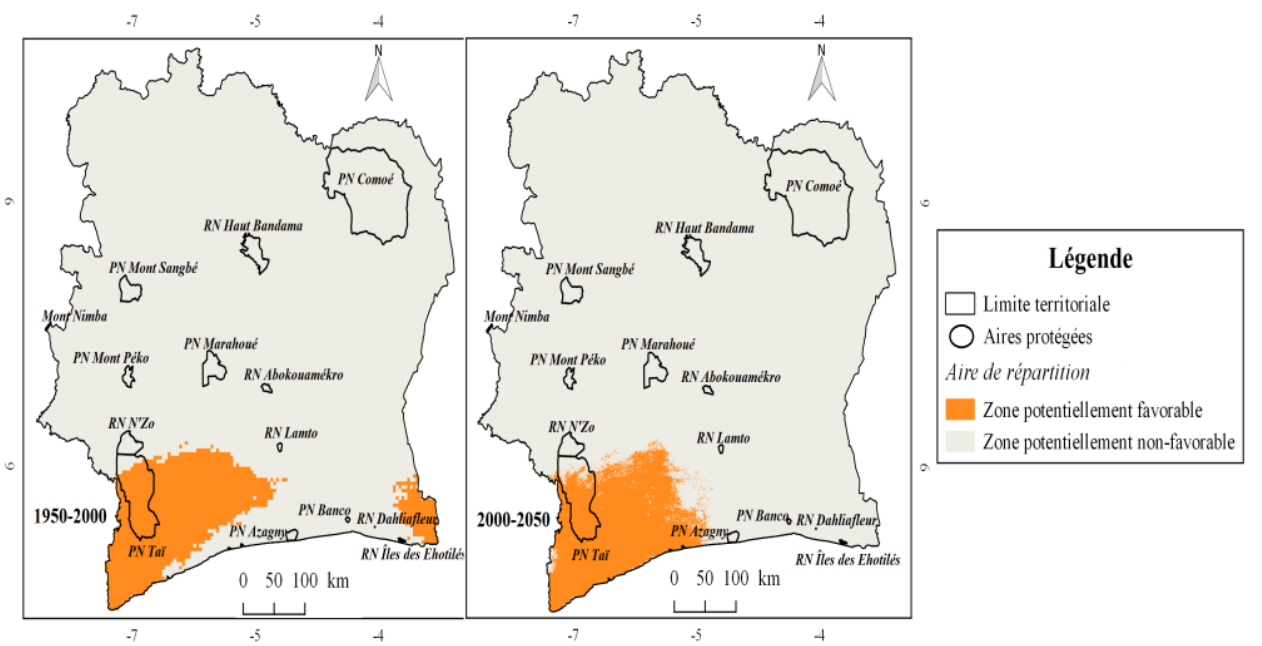

Figure 6: Distribution potentielle actuelle et actuelle et future de Elytraria ivorensis Dokosi en Côte d'Ivoire. 


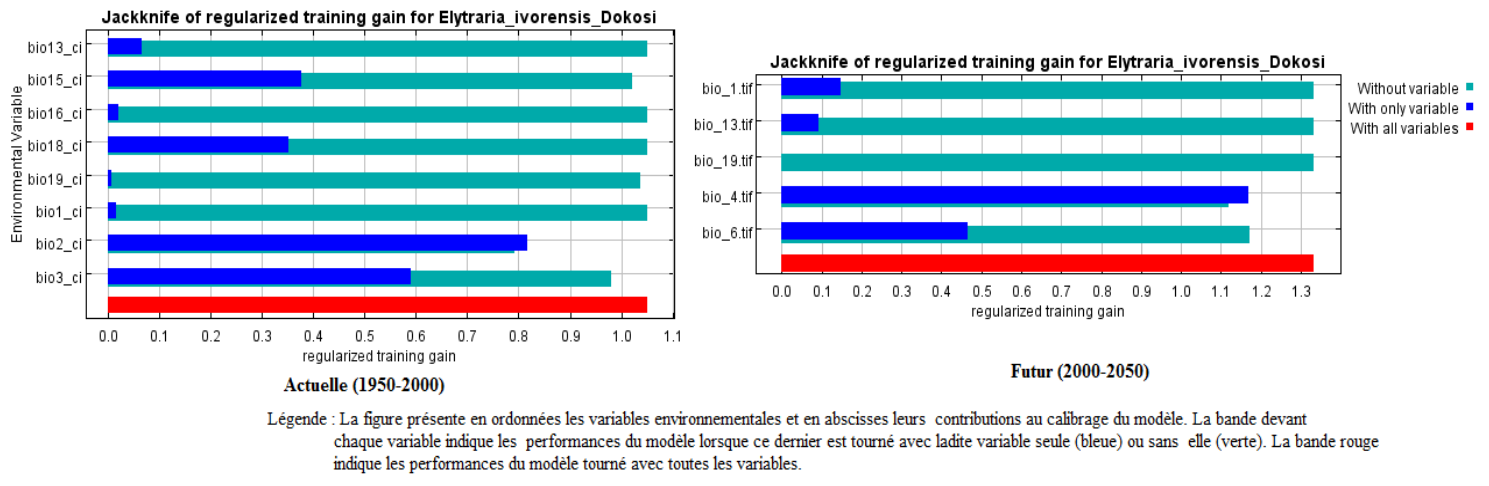

Figure 7: Résultat du test de Jackniffe sur les variables bioclimatiques du modèle.

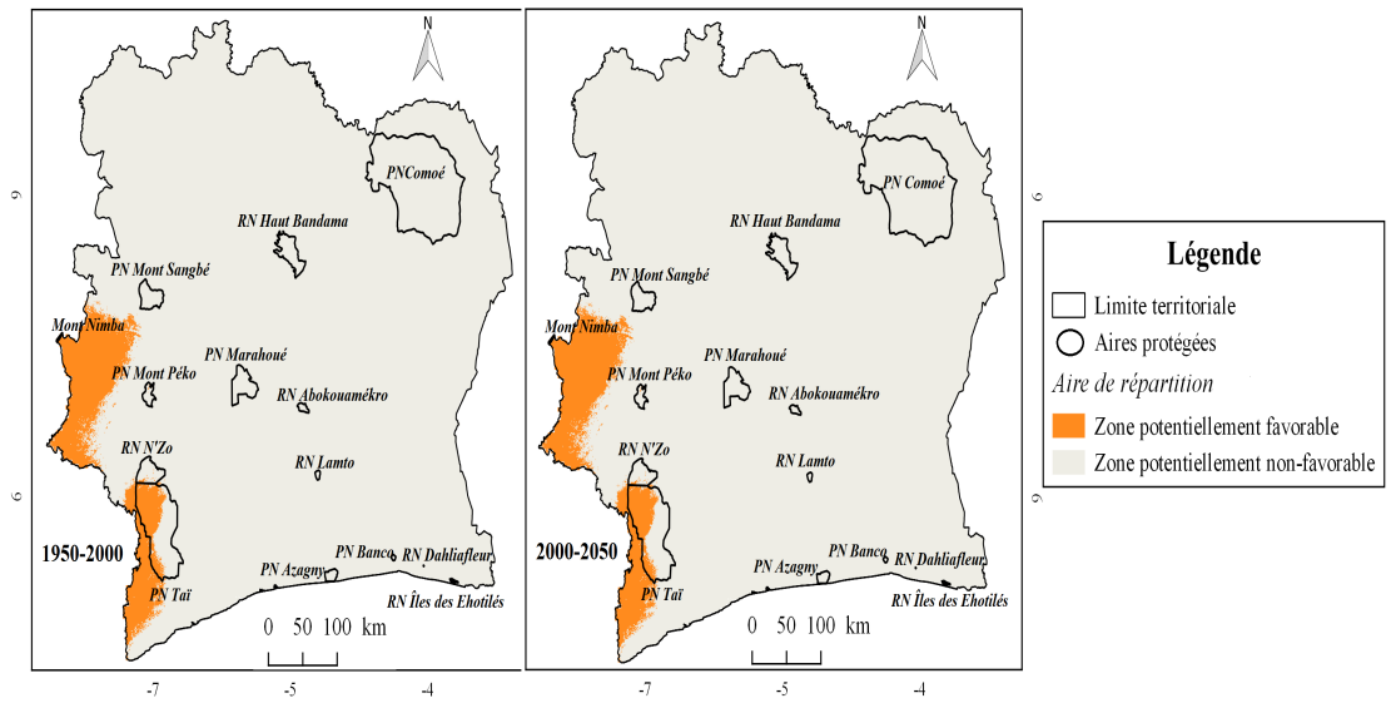

Figure 8: Distribution potentielle actuelle et future de Whitfieldia lateritia Hook en Côte d'Ivoire.

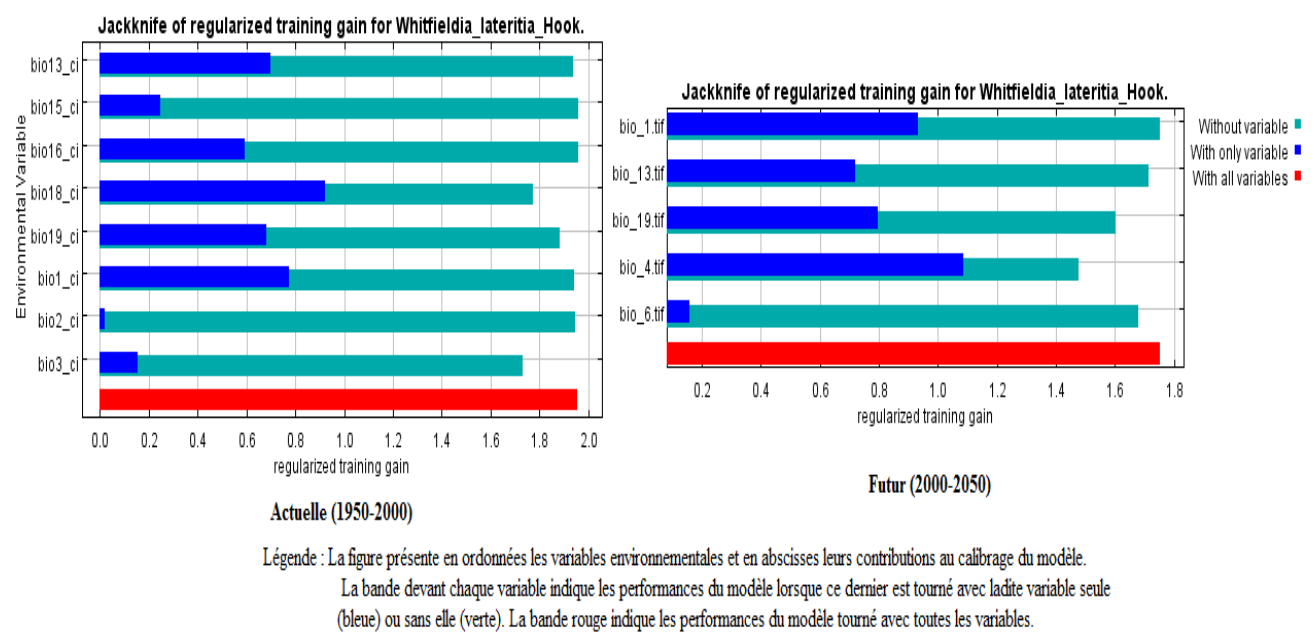

Figure 9: Résultat du test de Jackniffe sur les variables bioclimatiques du modèle. 


\section{DISCUSSION}

Diversité des Acanthaceae en Côte d'Ivoire

Le nombre d'espèces (106) et de genres (39) obtenu dans cette étude est différent de celui de Aké-Assi (2002) (85 espèces et 35 genres). Cette différence est due d'une part, à la prise en compte de notre part, de toutes les espèces sans distinction de leur appartenance ou non à la flore naturelle de Côte d'Ivoire. D'autre part à la compilation d'informations issues de plusieurs sources de données. En comparant sur le plan richesse taxonomique notre flore avec celle de l'Afrique centrale, nous constatons qu'elle est plus riche que la nôtre avec 310 espèces et 48 genres recensés obtenu par Koffi (2008). Cela s'explique par le fait que les travaux de Koffi (2008) ont été réalisés dans une vaste zone phytogéographique incluant la République Démocratique du Congo, le Rwanda et le Burundi et représente la diversité des Acanthaceae d'une région tandis que la présente étude été est réalisée dans une zone plus restreinte. La dominance des phanérophytes et des espèces de type GuinéoCongolaise (GC) dans le cortège floristique indiquerait qu'une grande partie des Acanthaceae a une préférence pour le secteur ombrophile. La proportion relativement élevée d'espèces introduites $(16 \%)$ confirme la classification de cette famille parmi les plus riches en plantes ornementales par Aké-Assi (2015) en Côte d'Ivoire. L'auteur signale d'ailleurs que les plantes introduites occupent une place de choix dans la flore ornementale ivoirienne. La diversité observée au sein de cette famille se traduit par la présence d'espèces à statut particulier et d'espèces endémiques

\section{Qualités du modèle et contribution des variables environnementales}

Les valeurs de l'AUC de l'ordre de 0,90 indiquent une excellente qualité des modèles générés pour la distribution des espèces modèles. Ceci indique la robustesse de la modélisation effectuée et atteste de l'excellente performance de l'algorithme MaxEnt à prédire les différents habitats de Dicliptera alternans Lindau, Elytraria ivorensis Dokosi et Whitfieldia lateritia Hook. Toutefois, il importe de rester vigilant avec ces résultats, puisque les facteurs écologiques et variables édaphiques n'ont pas été intégrés dans le modèle. En dépit de ces difficultés évoquées, Shmueli (2010) affirme qu'elles sont d'ordre mineur tant que l'objectif demeure la prédiction et non l'explication de la niche d'une espèce. De plus Fandohan et al. (2015), indique que le modèle MaxEnt ne constitue pas de problèmes lorsque la zone d'étude couvre une échelle réduite et que l'aire de référence pour tester et calibrer le modèle est limitée aux conditions climatiques où l'espèce est sous l'influence de mêmes facteurs climatiques. Les résultats révèlent que la «Saisonnalité de la température (Bio 4) » est la variable environnementale qui a le plus contribué à la prédiction du modèle des espèces Dicliptera alternans Lindau, Elytraria ivorensis Dokosi et Whitfieldia lateritia Hook. à l'horizon 2050. Cela suggère que la distribution future de ces espèces sera principalement influencée par les variations des températures.

\section{Aire de distribution potentielle des espèces}

Selon les résultats de nos travaux, les zones de distribution potentiellement favorables de nos espèces modèles actuelles et futur se retrouve à l'intérieur et aux alentours des aires protégées. Cela montre que le territoire national, à travers son réseau d'aires protégées, présente des conditions actuelles et futures favorables à la distribution des Acanthaceae. Ils confirment de ce fait que les aires protégées constituent des refuges qui favorisent encore la conservation de la biodiversité (Houinato et al., 2001) notamment des espèces endémiques et internationalement ou localement en danger face aux effets du changement climatique à l'horizon 2050. Cependant, les projections à l'horizon 2050 indiquent une régression de la superficie des zones potentiellement favorables des espèces Dicliptera alternans Lindau, Elytraria ivorensis Dokosi et Whitfieldia lateritia Hook. Cette régression s'expliquerait principalement par la projection de la hausse de la température et de la baisse 
du niveau des précipitations au niveau de la Côte d'ivoire (Goula et al., 2006; Oga et al., 2016). Ces résultats sont en concordances avec de nombreuses études qui mettent en évidence la régression des zones favorables à la distribution de certaines espèces (Boko et al., 2007 ; Houessou et al., 2012 ; Gbesso et al., 2013 ; Fandohan et al., 2015). De ce fait l'hypothèse selon laquelle les changements climatiques pourraient modifier l'aire de répartition des espèces semble être confirmée. Selon WWF (2011), la hausse des températures et la baisse des précipitations seraient le résultat de l'accroissement des concentrations des rejets anthropiques de Gaz à Effet de Serre (GES) fortement liées à la régression des forêts denses sempervirentes. Cette régression des forêts constatés en Côte d'Ivoire à l'instar des pays de la zone tropicale imputable aux pressions anthropiques, entraine une perte des habitats naturels favorables aux espèces telles que observée dans nos résultats. La régression des zones de distribution potentiellement favorables des espèces se fait par conversion en zone potentiellement non favorables induisant un accroissement de leur superficie. Le scénario A2 met l'accent sur un développement économique axé sur une population à fort taux de croissance avec un faible niveau technologique et de développement. Ainsi face aux besoins de cette population en expansion, on assistera de plus en plus à une conversion des forêts en zones agricoles. Ce serait l'une des raisons intervenant dans l'accroissement des zones non favorables à nos espèces. De plus Davis et al. (1986) estimaient déjà que 60000 espèces végétales disparaîtront d'ici 2050 si les tendances actuelles se maintiennent. Ces observations montrent que nos espèces verraient une régression probable de la superficie de leur zone favorable de distribution à l'horizon 2050 sous l'effet combiné de la dégradation des espaces forestiers et des variations climatiques.

\section{Conclusion}

Cette étude est une contribution à l'amélioration des connaissances de la biodiversité et de l'évaluation de l'impact des changements climatiques au niveau de la Côte d'Ivoire par le biais de la famille des Acanthaceae. A cet effet, elle a porté sur l'analyse, de la diversité et la distribution potentielle de quelques espèces des Acanthaceae présentes dans les herbiers de Côte d'Ivoire. La richesse de cette famille est estimée à 106 espèces regroupées en 39 genres parmi lesquelles 16 espèces endémiques et 7 espèces figurant sur la liste locale des espèces menacées et rares ivoiriennes de Aké-Assi (1998). Les Acanthaceae sont généralement des nanophanérophytes de type GuinéoCongolaise avec une proportion relativement élevée d'espèces introduites. La faible valeur du coefficient générique indique une bonne diversité au sein de cette famille. Comme la plupart des pays de la zone tropicale, la Côte d'Ivoire est sujette au changement climatique. Cette étude menée sur la distribution des espèces précisément sur celles des Acanthaceae, illustre bien ce phénomène et donne une idée de son impact dans la distribution spatiale des espèces. Il ressort de cette étude, qu'à l'horizon 2050, l'effet des changements climatiques couplée à la dégradation du couvert végétal entraineront une diminution importante de la surface des zones potentiellement favorables de distribution des espèces Dicliptera alternans Lindau, Elytraria ivorensis Dokosi et Whitfieldia lateritia Hook. respectivement de $67,66 \%$ (soit une perte de 26459,04 $\mathrm{km}^{2}$ ), $3,35 \%$ (soit une perte de $2120,85 \mathrm{~km}^{2}$ ) et $1,17 \%$ (soit une perte de $151,78 \mathrm{~km}^{2}$ ). Par contre, des accroissements des zones potentiellement non-favorables relativement seront observés pour ces espèces. Cependant, malgré ces changements observés, les résultats montrent que les conditions environnementales en Côte d'Ivoire demeureront favorables à la distribution des Acanthaceae surtout dans le réseau d'aires protégées. Ces travaux permettent ainsi d'évaluer l'importance de la distribution spatiotemporelle des espèces des populations locales et leur vulnérabilité face aux effets des changements climatiques. Ils permettront une meilleure orientation des prises de décision en matière de conservation et de gestions des 
zones de refuges de la biodiversité que sont les aires protégées.

\section{CONFLITS D'INTERETS}

Les auteurs déclarent qu'ils n'ont aucun conflit d'intérêts.

\section{CONTRIBUTIONS DES AUTEURS}

EEA est l'investigateur principal du travail. KJK et EA-A ont intervenus dans l'analyse des données et pour les nombreuses relectures du manuscrit.

\section{REMERCIEMENTS}

Les auteurs remercient vivement le centre National de Floristique (CNF), l'Institut Botanique Aké-Assi d'Andokoi (IBAAN) le Centre Suisse de Recherche Scientifique (CSRS) et le Global Biodiversity Information Facility (GBIF), qui nous ont permis d'avoir notre base de données. Ils remercient également tous les lecteurs qui ont contribué à l'amélioration de la qualité scientifique du manuscrit.

\section{REFERENCES}

Aké-Assi E. 2015. Plantes à potentialité décorative de la flore du sud de la Côte d'Ivoire : études taxinomique, ethnobotanique et essai de domestication de Thunbergia atacorensis Akoegninou et Lisowski (Acanthaceae), une espèce nouvellement introduite. Thèse de Doctorat, UFR Biosciences, Université Félix HOUPHOUEËT-BOIGNY, Côte d'Ivoire, $207 \mathrm{p}$.

Aké-Assi E, Koffi N'G, Tetchi NA. 2010. Atlas de la Biodiversité de l'Afrique de l'Ouest (Tome III). Atlas : Côte d'Ivoire; 324-325.

Aké-Assi L. 1984. Flore de la Côte d'Ivoire. Etude descriptive et biogéographique, avec quelques notes ethnobotaniques. Thèse Doctorat, Université Nationale d'Abidjan, Côte d'Ivoire, $1206 \mathrm{p}$.

Aké-Assi L. 1998. Impact de l'exploitation forestière et du développement agricole sur la conservation de la biodiversité biologique en Côte d'Ivoire. Le Flamboyant, (46) : 20-21.

Aké-Assi L. 2001. Flore de la Côte d'Ivoire 1, catalogue, systématique, biogéographie et écologie. Conservatoire et Jardin Botanique de Genève: Suisse- Boisseria ; 57: 396p.

Aké-Assi L. 2002. Flore de la Côte d'Ivoire 2, catalogue, systématique, biogéographie et écologie. Conservatoire et Jardin Botanique de Genève : Suisse Boisseria ; 58: 441p.

APG IV. 2016. An update of the Angiosperm Phylogeny Group classification for the orders and families of flowering plants. APG IV.

Araújo MB, Pearson RG, Thuiller W, Erhard M. 2005. Validation of species climate impact models under climate change. Global Change Biology, 11: 1504 -1513. DOI: $\quad$ https://doi.org/10.1111/j.13652486.2005.01000.x

Boko M, Niang I, Nyong A, Vogel C, Githeko A, Medany M, Osman-Elasha B, Tabo R, Yanda P. 2007. Africa. Climate Change 2007: Impacts, Adaptation and Vulnerability. Contribution of Working Group II to the Fourth Assessment Report of the Intergovernmental Panel on Climate Change. Parry ML, CanzianiOF, Palutikof JP, van der Linden PJ, Hanson CE (eds). Cambridge UK, Cambridge University Press : Cambridge ; 433-467.

Condé A. 2017. Impact des variations climatiques sur la distribution des principales espèces végétales dont les fruits sont consommés par les chimpanzés dans Le Parc National De Taï, Côte D'Ivoire. Mémoire de Master de Biodiversité et Valorisation des Écosystèmes de l'Université Félix Houphouët-Boigny, Côte D'Ivoire, 56 p.

Davis SD, Droop SJM, Gregerson P, Henson, Leon CJ, Villa-Lobos JL, Synge LH, Zantovska J. 1986. Plants in Danger. What do we Know? IUCN: Gland Switzerland and Cambridge U.K. F.; 520p.

Fandohan B, Gouwakinnou G, Fonton NH, Sinsin B, Liu J. 2013. Impact des changements climatiques sur la répartition géographique des aires favorables à la culture et à la conservation des fruitiers sous-utilisés : 
cas du tamarinier au Bénin. Biotechnol. Agron. Soc. Environ., 17(3): 450-462. DOI: https://popups.uliege.be:443/17804507/index.php?id=10186.

Fandohan AB, Moutouama JK, Samadori S, Biaou H, Gouwakinnou GN, Adomou CA. 2015. Le réseau d'aires protégées Bénin-Togo assure-t-il la conservation de Thunbergia atacorensis (Acanthaceae)? Rev. CAMES, 03(02).

Flato GM, Boer GJ, Lee WG, McFarlane NA, Ramsden D, Reader MC. 2000. The Canadian Centre for Climate Modelling and Analysis Global Coupled Model and its climate. Clim Dyn., 16:451-467. DOI: 10.1007/s003820050339

Gbesso FHG, Tente BHA, Gouwakinnou NG, Sinsin BA. 2013. Influence des changements climatiques sur la distribution géographique de Chrysophyllum albidum G. Don (Sapotaceae) au Benin. Int. J. Biol. Chem. Sci., 7(5): 2007-2018. DOI: http://dx.doi.org/10.4314/ijbcs.v7i5.18

GIEC. 2007. Groupe d'Experts Intergouvernemental sur l'Evolution du Climat, Bilan 2007 des changements climatiques. Contribution des Groupes de travail I, II et III au quatrième Rapport d'évaluation du Groupe d'experts intergouvernemental sur l'évolution du climat, Genève-Suisse, $103 \mathrm{p}$.

Gnagbo A. 2015. Diversité, distribution et utilisations des épiphytes vasculaires des strates inférieures des forêts côtières de Côte D'ivoire : Cas du Parc National d'Azagny. Thèse de doctorat en écologie végétale, UFR Biosciences, Université Félix Houfouët Boigny, Abidjan, Côte d'Ivoire, $216 \mathrm{p}$.

Gnagbo A, Kpangui KB, Adou YCY. 2015. Distribution des épiphytes de Côte d'Ivoire: effets des zones phytogéographiques et des variations pluviométriques. Afrique Science, 11(1): 175-186.

Guarino L, Jarvis A, Hijmans RJ, Maxted N. 2002. Geographic Information Systems (GIS) and the Conservation and Use of Plant Genetic Resources. In: Managing plant genetic diversity, Engels JMM, Ramanatha RV, Brown AHD, Jacson MT(éd). International Plant Genetic Resources Institute (IPGRI) Rome: Italie; 387-404.

Grubben GJH. 2004. Ressources végétales d'Afrique Tropicale 2. Légumes Fondation Prota Wageningen : Pays Bas/ Backhuys ; 737p.

Gnagbo A. 2015. Diversité, distribution et utilisations des épiphytes vasculaires des strates inférieures des forêts côtières de Côte D'ivoire : Cas du Parc National d'Azagny. Thèse de doctorat en écologie végétale, UFR Biosciences, Université Félix Houfouët Boigny, Abidjan-Côte d'Ivoire, 216 p.

Houessou GL, Lougbegnon OT, Gbesso GHF, Anagonou SEL, Sinsin B. 2012. Ethnobotanical study of the African star apple (Chrysophyllum albidum G. Don) in the Southern Benin (West Africa). Journal of Ethnobiology and Ethnomedicine, 8: $40 . \quad$ DOI: https://doi.org/10.1186/1746-4269-8-40

Houinato M, Sinsin B, Lejoly J. 2001. Impact des feux de brousse sur la dynamique des communautés végétales dans la forêt de Bassila (Bénin). Acta Bot. Gall., 148(3): 237-251.

DOI: 10.1080/12538078.2001.10515891

IPCC. 2007. Climate Change. Synthesis Report. Contribution of Working Groups I, II \& III to the Fourth Assessment Report of the Intergovernmental Panel on Climate Change. Geneva Cambridge University Press Cambridge: UK and New York-USA; 339-378. DOI: 10.1017/CBO9780511546013.

Koffi KJ, Champluvier D, Danho FRN, De Cannière C, Traoré D, Lejoly J, Robbrecht E, Bogaert J. 2008. Analyse de la distribution spatiale des Acanthaceae en Afrique Centrale et comparaison avec les théories phytogéographiques de Robyns, White et Ndjele. Sciences et Nature, 5(2): 101110.

DOI : https://www.ajol.info/index.php/ scinat/article/view/42156/9284. 
Koffi KJ. 2008. Analyse des structures spatiales des données de distribution phytogéographique des Acanthaceae en Afrique centrale. Thèse de Doctorat Université Libre de Bruxelles, Faculté des sciences, Ecole Interfacultaire de Bioingenieurs, Service d'Ecologie du paysage et Systèmes de production végétale, Bruxelles, 240p.

Kouakou E, Goula BTA, Kouassi AM. 2012. Analyze of climate variability and change impacts on hydro-climate parameters: case study of Côte d'Ivoire. International Journal of Scientific \& Engineering Research, 3(2): 1-8.

Kouamé YM, Soro GE, Kouakou KE, Kouadio ZA, N'Diaye MEH, Goula BTA, Issiaka S. 2014. Scenarios des changements climatiques pour les précipitations et les températures en Afrique subsaharienne tropicale humide : cas du bassin versant de Davo, Côte d'Ivoire. Larhyss Journal, 18: 197-213.

N'Guessan K, Annick K, Stefan P, Dossahoua T, Aké-Assi L. 2009. La biodiversité en Côte d'Ivoire: Etat des lieux et facteurs de menace, 273p.

Oga YMS, Adja M, Yapi AF, Kpan JG, Baka D, Yao KT, Biémi J. 2016. Projection de la variabilité climatique à l'horizon 2050 dans la zone côtière au Sud-Est de la Côte d'Ivoire (d'Abidjan à Aboisso). Larhyss Journal, 25: 67-81.

Phillips SJ, Anderson RP, Schapire RE.2006. Maximum entropy modeling of species geographic distributions. Ecol. Model., 190: 231-259.

DOI:

10.1016/j.ecolmodel.2005.03.026

Sala E, Ballesteros E, Starr RM. 2001. Rapid decline of Nassau grouper spawning aggregations in Belize: Fishery Management and Conservation Needs, Fisheries, 26(10): 23-30. DOI: 10.1577/1548-8446(2001)026<0023: RDONGS > 2.0.CO; 2.
Shmueli G. 2010. To explain or to predict? Statistical Science, 25(3): 289-310. DOI: 10.1214/10-STS330

Sorokoby VM, Saley MB, Kouamé KF, Djagoua EMV, Affian K, Biemi J. 2013. Variabilité spatio-temporelle des paramètres climatiques et son incidence sur le tarrissement dans les bassins versants du Bô et Debo (département de Soubré au Sud-Ouest de la Côte d'Ivoire). International Journal of Innovation and Applied Studies, 2(3): 287-299.

Toyi MS, Barima YSS, Mama A, André M, Bastin J-F, De Cannière C, Sinsin B, Bogaert J. 2013. Tree plantation will not compensate natural woody vegetation cover loss in the Atlantic Department of Southern Benin. Tropicultura, 31(1): 6270.

Tuo FN, Kouao JK, Kouassi FA, Kone M, Bakayoko A, Bogaert J. 2017. Etude de la diversité, de l'endémisme et de la distribution spatiale des Rubiaceae de Côte d'Ivoire. Int. J. Biol. Chem. Sci., 11(2): 777-797. DOI: https://dx.doi.org/10.4314/ijbcs.v11i2.20

UICN. 2016. The IUCN Red List of Threatened Species. UICN. www.iucnredlist.org.

Vroh BTA, Adou YCY, Kpangui KB, Goné BZB, Kouamé D, Koffi JK. 2016. Comparing Suitable Habitat Models to Predict Rare and Endemic Plant Species Distributions: What are the Limits of the Niche of Cola lorougnonis (Malvaceae) in Côte d'Ivoire? Environment and Natural Resources Research, 6(3): 1-17. DOI:10.5539/enrr.v6n3p1

Williams JW, Jackson ST, Kutsbach JE. 2007. Projected distribution of novel and disappearing climates by 2100 AD. Proc. Natl. Acad. Sci. U.S.A., 104: 5738-5742.

WWF (World wide fund for nature). 2011. Rapport des impacts du changement climatiques sur les espèces. WWF, 21p. 